\title{
Chinese herbal medicine Buyang Huanwu Decoction combined with acupuncture to treat sequela of apoplexy: a meta-analysis of randomized controlled trials
}

\author{
Meng-Jie Zeng ${ }^{1 \wedge}$, Lin-Jun $\mathrm{Li}^{2}$, Zhi-Quan $\mathrm{Wu}^{3 \wedge}$ \\ ${ }^{1}$ School of Traditional Chinese Medicine, Macao University of Science and Technology, Macao, China; ${ }^{2}$ Department of Acupuncture and Massage, \\ Yunnan University of Traditional Chinese Medicine, Kunming, China; ${ }^{3}$ Department of Rehabilitation Medicine, Sanya Central Hospital, Sanya, China \\ Contributions: (I) Conception and design: MJ Zeng, LJ Li; (II) Administrative support: ZQ Wu; (III) Provision of study materials or patients: ZQ Wu; \\ (IV) Collection and assembly of data: MJ Zeng, LJ Li; (V) Data analysis and interpretation: MJ Zeng; (VI) Manuscript writing: All authors; (VII) \\ Final approval of manuscript: All authors. \\ Correspondence to: Zhi-Quan Wu. Department of Rehabilitation Medicine, Sanya Central Hospital, No.146 Jiefang 4th Road, Tianya District, Sanya \\ 572000, China. Email: 513376829@qq.com.
}

Background Buyang Huanwu Decoction combined with acupuncture has a wide range of applications
in the treatment of stroke sequelae, and there are many clinical trial reports. We systematically evaluated
the clinical efficacy and safety of Buyang Huanwu Decoction combined with acupuncture in treating stroke
sequelae, and evaluated overall research quality.
Methods: We searched 7 databases which includes the Chinese Journal Full-text Database (CNKI),
VIP Database (VIP), China Biomedicine (SinoMed), Wanfang Database and PubMed, Cochrane Central,
EMBASE from January 2010 to December 2019 Literature references. We selected randomized controlled
trials that tested the effects of Buyang Huanwu Decoction combined with acupuncture on stroke sequelae.
The authors extracted data and independently assessed quality. We used RevMan 5.3.0 software to analyze
the data of randomized trials.

Results: A total of 7 articles were identified, including 902 patients. The overall quality of the included trials was poor, and one of them was moderate. Meta-analysis results showed that the experimental group of Buyang Huanwu decoction combined with acupuncture treatment of stroke sequela compared with the control group clearly improved the clinical efficacy improved the clinical efficacy rate (RR 1.18, 95\% CI: 1.12 , 1.25), $\mathrm{P}<0.00001]$. Among them, three trials included the incidence of adverse reactions (RR $0.22,95 \% \mathrm{CI}$ : $0.09,0.52, \mathrm{P}=0.0006$ ), which also confirmed the safety of its treatment.

Conclusions: Buyang Huanwu Decoction combined with acupuncture is an effective therapy to ameliorate the clinical symptoms of stroke sequelae. In order to further determine the effectiveness and safety of Buyang Huanwu Decoction combined with acupuncture in treating stroke sequelae, more rigorous design, multicenter and prospective RCT must be carried out.

Keywords: Chinese herbal medicine; Buyang Huanwu Decoction; acupuncture; sequela of apoplexy; metaanalysis of randomized controlled trials

Submitted May 09, 2020. Accepted for publication Oct 11, 2020.

doi: 10.21037/apm-20-1092

View this article at: http://dx.doi.org/10.21037/apm-20-1092

^ ORCID: Meng-Jie Zeng, 0000-0002-7751-7177; Zhi-Quan Wu, 0000-0002-8519-6473. 


\section{Introduction}

Stroke is a common disease in neurology, patients often have different degrees of sequelae, which affect the patient's quality of life (1). Stroke is currently the leading cause of death and disability in our population, and about 2 million strokes occur every year in China (2). The post-stroke sequelae mainly refer to a condition left after hemorrhage of acute cerebrovascular disease, hemiplegia, numbness, skewed eyes, and poor speech. At present, stroke, cancer, and coronary heart disease have become the three major diseases of human death in the world, which poses a serious threat to the safety of patients' lives and physical and mental health (3). In recent years, more and more reports have shown that acupuncture combined with traditional Chinese medicine has a significant effect in the treatment of sequelae of stroke, which can improve the patient's nerve function and limb function, relieve the symptoms of sequelae, and improve the quality of life of patients (4).

In China, traditional Chinese medicine and acupuncture are commonly used to treat sequelae of stroke. Buyang Huanwu Decoction is a common prescription which can treat sequelae of stroke. Buyang Huanwu Decoction is the prescription of "Medical Forest Correction" by Qing-ren Wang, a famous doctor in the Qing Dynasty. It consists of astragalus, angelica tail, red peony root, earthworm, Chuanxiong, safflower, and peach kernel (5). Its traditional function is to replenish qi, activate blood circulation and remove blood stasis. Through the analysis of the names of diseases treated by Buyang Huanwu Decoction and the bibliometrics research of modern literature, it is found that the most frequent TCM diseases are apoplectic sequelae. At the same time, acupuncture combined with Buyang Huanwu Decoction is also common in clinical treatment of sequelae of stroke, and its curative effect is very good.

In order to scientifically assess effectiveness of Buyang Huanwu Decoction combined with acupuncture treated stroke sequelae, we conducted a comprehensive review of the medical literature and a meta-analysis of randomized controlled trials of Buyang Huanwu Decoction combined with acupuncture in the treatment of stroke sequelae. We present the following article in accordance with the PRISMA reporting checklist (available at http://dx.doi. org/10.21037/apm-20-1092).

\section{Methods}

\section{Search strategy}

Searched 3 English electronic databases and 4 Chinese electronic databases: China Biomedicine (SinoMed), VIP Database (VIP)and Wanfang Database, PubMed, Cochrane Central, EMBASE, Chinese Journal Full-text Database $(\mathrm{CNKI})$. The conference records and papers were retrieved from CNKI and Wanfang databases for unpublished trials. According to the search database, use the following search terms (or Chinese database equivalent to Chinese) are used: "Buyang Huanwu Decoction" "Acupuncture" "Buyang Huanwu Decoction combined with acupuncture treatment" "Buyang Huanwu Decoction plus Combined with acupuncture treatment" "stroke of sequelae" "stroke of apoplexy" and "random".

The specific search strategy of PubMed was as follows:

\#1 Search $(((()(((($ Sequelae of stroke [Title / Abstract] $)$ OR Sequela of apoplexy [Title / Abstract])

\#2 Search ((()(Buyang Huanwu Decoction [Title / Abstract]) OR Buyang Huanwu Decoction combined with acupuncture treatment [Title / Abstract]) OR Acupuncture [Title / Abstract]) OR Acupuncture and Moxibustion Treatment of Buyang Huanwu Decoction [Abstract/Title])

\#3 Search random

\#1 and \# 2 and \# and3.

\section{Eligibility criteria}

If these studies meet the following criteria, they are analyzed and studied: (I) the included studies are randomized controlled trials (RCT); (II) regarding the type of intervention, the participants in the treatment group were treated with Buyang Huanwu Decoction combined with acupuncture; (III) diagnosis after CT or MRI; (IV) the efficacy or curative effect of Buyang Huanwu Decoction combined with acupuncture on the treatment of sequelae of stroke, the main therapeutic indicators: clinical efficacy rate and adverse reaction rate.

Exclusion criteria include: (I) the treatment group adopts simple Western medicine treatment or single Chinese medicine treatment; (II) the details of the test are unclear, or the result data is incomplete; (III) the same test data is repeatedly published or animal tests; (IV) the test data is 
Table 1 The basic characteristics of the literature

\begin{tabular}{|c|c|c|c|c|c|}
\hline Author & $\begin{array}{c}\text { Article } \\
\text { publication time }\end{array}$ & $\begin{array}{l}\text { Sample } \\
\text { size }(T / C)\end{array}$ & \multicolumn{2}{|c|}{ Intervention } & $\begin{array}{l}\text { Outcome } \\
\text { indicators }\end{array}$ \\
\hline Lei et al. (6) & 2017 & $80 / 80$ & $\begin{array}{l}\text { Buyang Huanwu Decoction plus or minus } \\
\text { combined acupuncture routine treatment }\end{array}$ & Conventional treatment & (1) \\
\hline Lei et al. (7) & 2018 & $66 / 66$ & $\begin{array}{l}\text { Buyang Huanwu Decoction combined } \\
\text { acupuncture routine treatment }\end{array}$ & $\begin{array}{l}\text { Routine rehabilitation } \\
\text { treatment }\end{array}$ & (1) \\
\hline Shang (9) & 2014 & $75 / 75$ & $\begin{array}{l}\text { Buyang Huanwu Decoction plus or minus } \\
\text { combined acupuncture routine treatment }\end{array}$ & Acupuncture treatment & (1) \\
\hline $\begin{array}{l}\text { Song et al. } \\
\text { (10) }\end{array}$ & 2017 & $60 / 60$ & $\begin{array}{l}\text { Buyang Huanwu Decoction plus or minus } \\
\text { combined acupuncture routine treatment }\end{array}$ & Conventional treatment & (1) \\
\hline $\begin{array}{l}\text { He et al. } \\
\text { (12) }\end{array}$ & 2017 & $50 / 50$ & $\begin{array}{l}\text { Buyang Huanwu Decoction plus or minus } \\
\text { combined acupuncture routine treatment }\end{array}$ & Conventional treatment & (1)(2) \\
\hline
\end{tabular}

T: the experimental group; C: the control group; (1) : clinical efficacy rate; (2) : adverse reaction rate; conventional treatment: Western medicine.

obviously defective or incompletely provided, and the test protocol design is not reasonable; $(\mathrm{V})$ there were less than 50 patients in each literature.

\section{Statistical analysis}

An author (ZQW) screened the literature. Firstly, screen the titles and abstracts of all records to determine whether the full text of the relevant research meets the conditions. If there is a disagreement, it can be resolved through discussion with the lead author (MJZ). The clinical efficacy rate and adverse reaction rate were used to evaluate the improvement of clinical symptoms in the treatment of sequelae of stroke with Buyang Huanwu Decoction combined with acupuncture. The table describes in detail the basic characteristics of the literature (Table 1).

\section{Assessment of risk of bias}

The author (LJL) used the Cochrane bias risk tool to evaluate the quality of included trials (13). The following items were assessed: the following 7 aspects were assessed: (I) patient blindness; (II) random sequence generation; (III) evaluator blindness; (IV) Incomplete data; (V) selective reporting; (VI) allocation concealment; (VII) other sources of bias. According to the 7 criteria given, these assessment results are classified as low risk, unclear risk, or high risk. When judging other biases, we consider sample size estimates, exclusion criteria and inclusion (Figure 1). The differences were resolved through discussions with primary author (MJZ).

\section{Data analysis}

We use RevMan 5.3 software for meta-analysis. The heterogeneity of the results of each study was evaluated, and the $\chi^{2}$ test was used uniformly. If $\mathrm{I}^{2} \geq 50 \%$, it indicates low heterogeneity among study groups, and the fixed effect mode is adopted. Based on all the above analysis, the measurement data uses the mean difference (MD) or standardized mean difference (SMD) for effect combination, and the count data uses the odds ratio (OR) or relative risk (RR) combined effect influence volume. The inverted funnel chart was used to assess the risk of publication bias for each study.

\section{Results}

\section{Search results}

According to the search method, 155 articles were got from seven electronic databases. 55 duplicate clauses were removed. After preliminary screening of abstracts and titles, because they did not meet the research criteria, so 19 articles were precluded. At the same time, we searched 


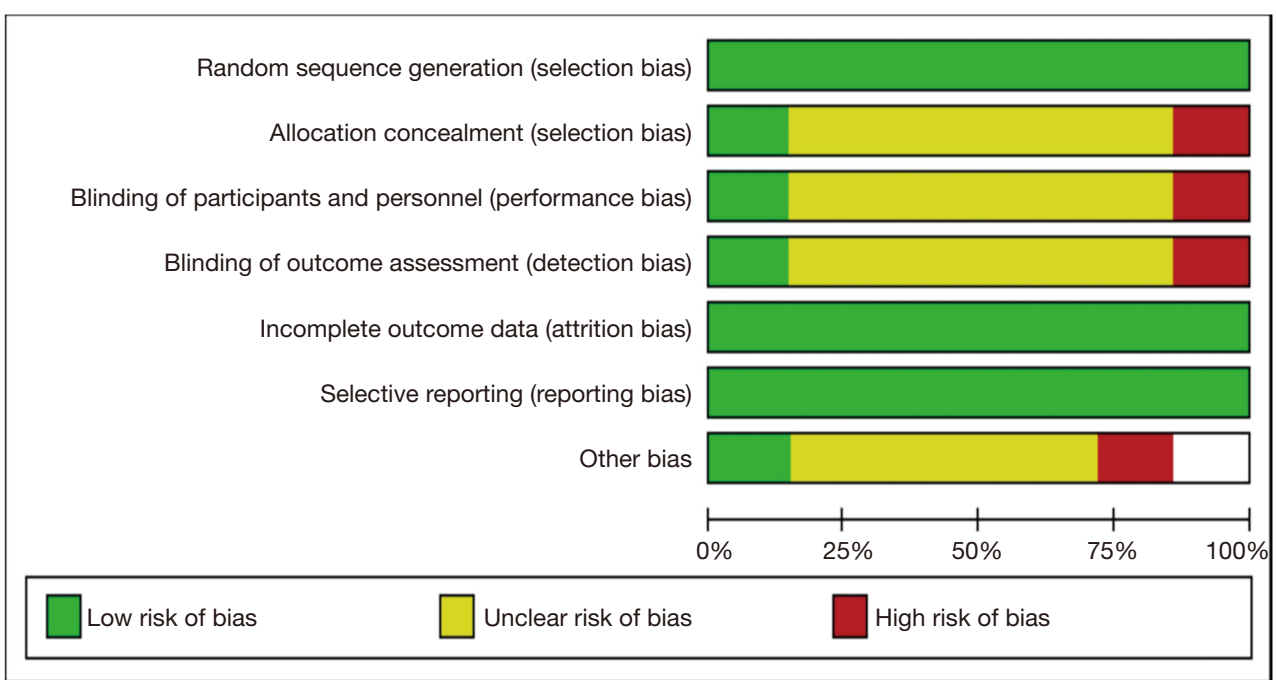

Figure 1 Assessment of risk of bias.

81 full-text studies to further determine the reasons for the deletion of 73 articles. Finally, seven articles participated in the meta-analysis (Figure 2).

\section{Study characteristics}

Table 1 summarizes the characteristics of 10 trials involving 902 participants. All included trials were conducted in China and published in Chinese. The sample size varied from 50 to 160 participants, with an average of 50 participants in each trial, and patients aged 40 to 90 years. All participants were diagnosed by clinical manifestations and imaging examination. The duration of treatment with RCT is 1 to 6 months, and the efficacy was assessed at the end of treatment.

There are two trials comparing Buyang Huanwu Decoction with acupuncture and western medicine conventional treatment, and two are comparing with aspirin; two of them are comparing with traditional Chinese medicine; the most special is comparing conventional treatment with traditional Chinese medicine injection. No trials used placebo or no intervention as a control. Buyang Huanwu Decoction is a decoction in all the included trials.

Among the 10 qualified RCTs, 7 trials passed the clinical effectiveness measurement and 3 trials were evaluated by the adverse reaction rate. Therefore, the main clinical effective rate and adverse reaction rate reflect the therapeutic effect of Buyang Huanwu Decoction combined with acupuncture on stroke sequelae.

\section{Continuous data results}

We conducted a comprehensive analysis of 902 patients. The results of the meta-analysis of clinical efficacy are shown on Figure 3. The $\chi^{2}$ test showed that $\mathrm{I}^{2}=0 \%(<50 \%)$, $\mathrm{P}=0.82$, indicating that there is no heterogeneity, so select the fixed effect model. It can be seen from the forest map that the total effective number of patients with stroke sequela using the treatment of Buyang Huanwu Decoction combined with acupuncture is significantly more than the control group (RR 1.18, $95 \%$ CI: 1.12, 1.25, P<0.00001; Figure 3).

Three trials involved studies of adverse reaction rates, and we used a random effects model for statistical analysis. Analysis was carried out according to different control groups. The comprehensive analysis results show that the meta-analysis results of the adverse reaction rate are shown in Figure 4. $\chi^{2}$ test shows that $\mathrm{I}^{2}=0 \%(<50 \%), \mathrm{P}=0.69$, indicating that there is no heterogeneity between the included documents, so the choice is fixed effect model. As can be seen from the forest map, the adverse reaction rate of the treatment of sequelae of stroke with Buyang Huanwu Decoction combined with acupuncture is significantly lower than that of the control group (RR 0.22, 95\% CI: 0.09, 0.52, $\mathrm{P}=0.0006$; Figure 4). 


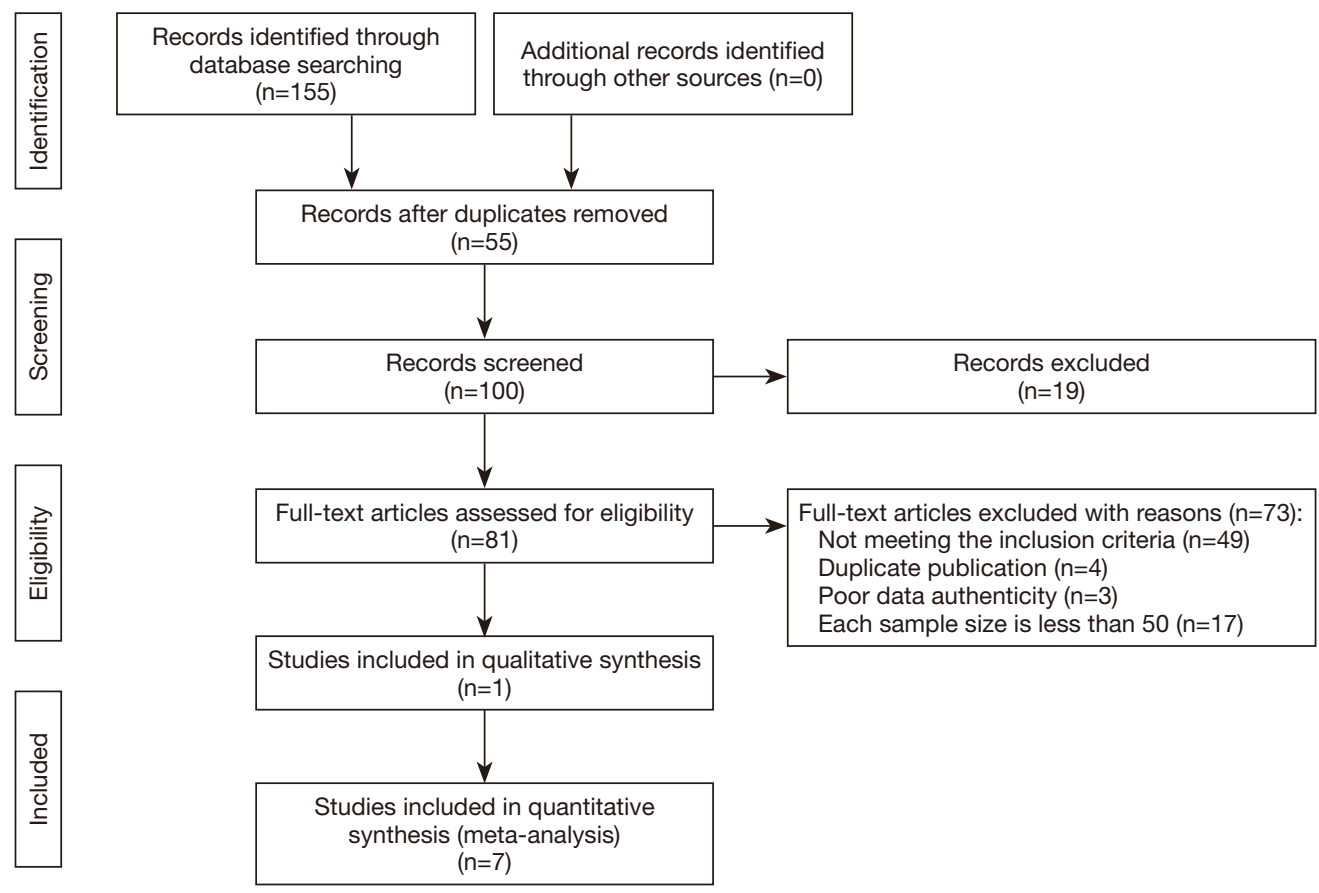

Figure 2 Study selection flow chart.

\begin{tabular}{|c|c|c|c|c|c|c|c|}
\hline \multirow{2}{*}{ Study or Subgroup } & \multicolumn{2}{|c|}{ Experimental } & \multicolumn{2}{|c|}{ Control } & \multirow{2}{*}{ Weight } & \multirow{2}{*}{$\begin{array}{c}\text { Risk Ratio } \\
\mathrm{M}-\mathrm{H}, \text { Fixed, } 95 \% \mathrm{Cl}\end{array}$} & \multirow{2}{*}{$\begin{array}{c}\text { Risk Ratio } \\
\mathrm{M}-\mathrm{H}, \text { Fixed, 95\% Cl }\end{array}$} \\
\hline & & & & & & & \\
\hline He Baojun 2017 & 46 & 50 & 38 & 50 & $10.7 \%$ & $1.21[1.02,1.44]$ & $\square$ \\
\hline Lei Huaping 2017 & 59 & 66 & 49 & 66 & $13.8 \%$ & $1.20[1.02,1.42]$ & $\longrightarrow$ \\
\hline Lei Zhaoming 2018 & 74 & 80 & 66 & 80 & $18.5 \%$ & $1.12[1.00,1.26]$ & $\because-$ \\
\hline Liu Xiwei 2019 & 57 & 60 & 46 & 60 & $12.9 \%$ & $1.24[1.07,1.44]$ & $\longrightarrow$ \\
\hline Shang Fake 2014 & 72 & 75 & 64 & 75 & $18.0 \%$ & $1.13[1.01,1.25]$ & - \\
\hline Song Xinan 2016 & 57 & 60 & 49 & 60 & $13.8 \%$ & $1.16[1.02,1.33]$ & $\rightarrow$ \\
\hline Zhang Rongxi 2016 & 56 & 60 & 44 & 60 & $12.4 \%$ & $1.27[1.08,1.50]$ & \\
\hline Total $(95 \% \mathrm{Cl})$ & & 451 & & 451 & $100.0 \%$ & $1.18[1.12,1.25]$ & 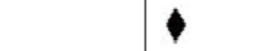 \\
\hline Total events & 421 & & 356 & & & & \\
\hline \multicolumn{7}{|c|}{ Heterogeneity: $\mathrm{Chi}^{2}=2.93, \mathrm{df}=6(\mathrm{P}=0.82) ; \mathrm{I}^{2}=0 \%$} & $\begin{array}{cccc}0.5 & 0.7 & 1 & 1.5 \\
& \text { Experimental } & \text { Control }\end{array}$ \\
\hline
\end{tabular}

Figure 3 Clinical efficacy.

\section{Funnel plot}

Seven reported clinical efficacy trials were analyzed using funnel plots to explore publication bias (Figure 5). The plot is symmetrical and there is no obvious deviation on the surface.

\section{Methodological quality}

All included trials were randomized trials in parallel groups, and no trial descriptions were assigned hidden or blind. We assessed the risk of bias through Cochrane's risk of bias. Detailed information based on the results is shown 


\begin{tabular}{|c|c|c|c|c|c|c|c|c|}
\hline Study or Subgroup & $\begin{array}{c}\text { Experimen } \\
\text { Events }\end{array}$ & al & $\begin{array}{l}\text { Cont } \\
\text { Events }\end{array}$ & Total & Weight & $\begin{array}{c}\text { Risk Ratio } \\
\text { M-H, Fixed, 95\% Cl }\end{array}$ & $\begin{array}{c}\text { Risk Ratio } \\
\text { M-H, Fixed, 95\% Cl }\end{array}$ & \\
\hline He Baojun 2017 & 2 & 50 & 6 & 50 & $22.2 \%$ & $0.33[0.07,1.57]$ & + & \\
\hline Liu Xiwei 2019 & 3 & 60 & 12 & 60 & $44.4 \%$ & $0.25[0.07,0.84]$ & & \\
\hline Zhang Rongxi 2016 & 1 & 60 & 9 & 60 & $33.3 \%$ & $0.11[0.01,0.85]$ & & \\
\hline Total $(95 \% \mathrm{Cl})$ & & 170 & & 170 & $100.0 \%$ & $0.22[0.09,0.52]$ & & \\
\hline Total events & 6 & & 27 & & & & & \\
\hline \multicolumn{6}{|c|}{ Heterogeneity: Chi $^{2}=0.74, d f=2(P=0.69) ; l^{2}=0 \%$} & 0.001 & 110 & 1000 \\
\hline
\end{tabular}

Figure 4 Adverse reaction rate.

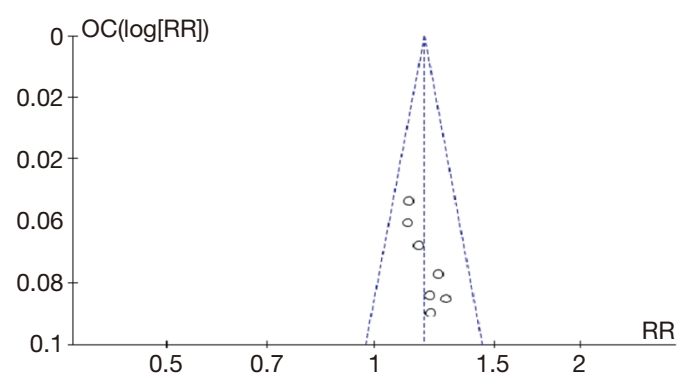

Figure 5 Funnel plot.

in Figure 6. The overall quality of the included trials was poor, and two of them were moderate. All RCTs contain the randomization principle, and no RCT describes allocation concealment or blindness by evaluators.

\section{Discussion}

\section{Summary of findings}

With the acceleration of social aging, the incidence of stroke increases year by year, prompt and effective treatment is of great significance for reducing the mortality and sequelae (14). The herbs are not expensive and will not increase the financial burden of the patient. In our study, the systematic analysis confirmed the clinical efficacy and safety of the treatment of Buyang Huanwu Decoction combined with acupuncture on stroke sequelae. The adverse reaction rates are less likely to occur during Buyang Huanwu Decoction combined with acupuncture.

\section{Strengths and limitations}

This study has some limitations. Most trials did not report

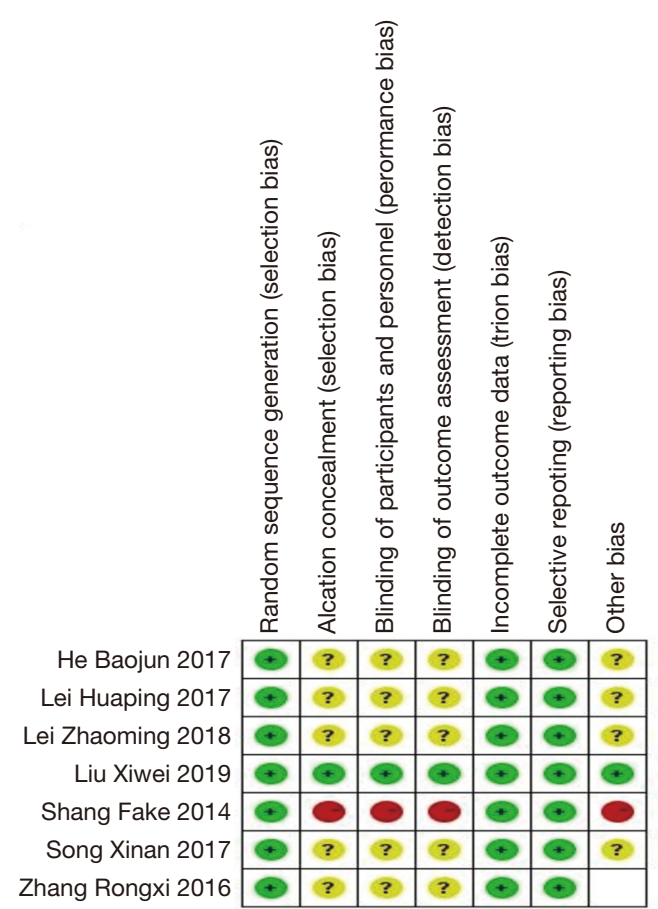

Figure 6 Risk of bias in the included randomized controlled trials.

randomization. The methodological quality of tests is usually poor. and all trials lacked blinding information. In addition, sample size and intention analysis estimation were not conducted. There may be publication bias because of the insufficient number of mutual trials in the meta-analysis (15). Due to a large number of unexplained heterogeneities, the performance of some meta-analysis is limited. We tried to compare Buyang Huanwu Decoction with other proprietary Chinese medicines, but due to the limited number of such trials, we were unable to draw any 
conclusions.

\section{Implications for further study}

In future research, we will focus on three main issues that should be considered in traditional Chinese medicine study: (I) the sample size should need to be based on sufficient statistical data, and the calculation method of the sample size method should be reported in the text; the random method needs to be fully described and appropriately hidden; (II) some drugs with obvious therapeutic effects and some ineffective or harmful conventional drugs should be recorded in detail; (III) future clinical trials should pay attention to the occurrence of more adverse events, especially for long-term treatment safety studies. Adverse events should adopt international standards Medical terms are recorded and reported.

\section{Conclusions}

The results of the test show that it is more effective, and it is certain that Buyang Huanwu Decoction combined with acupuncture is effective in treating the sequelae of stroke. However, because of the poor quality of this method, its effectiveness cannot be determined. We suggest conducting more meticulously, designed large sample clinical trials, which can reduce the one sidedness, heterogeneity, and contingency, of various studies in a single article. It can be known that long-term Buyang Huanwu decoction combined with acupuncture has less adverse reaction rate than conventional western medicine treatment.

\section{Acknowledgments}

Funding: This work was supported by Sanya Medical Science and Technology Innovation Project (No. 2017YW06).

\section{Footnote}

Reporting Checklist: The authors have completed the PRISMA reporting checklist. Available at http://dx.doi. org/10.21037/apm-20-1092

Conflicts of Interest: All authors have completed the ICMJE uniform disclosure form (available at http://dx.doi. org/10.21037/apm-20-1092). The authors have no conflicts of interest to declare.
Ethical Statement: The authors are accountable for all aspects of the work in ensuring that questions related to the accuracy or integrity of any part of the work are appropriately investigated and resolved.

Open Access Statement: This is an Open Access article distributed in accordance with the Creative Commons Attribution-NonCommercial-NoDerivs 4.0 International License (CC BY-NC-ND 4.0), which permits the noncommercial replication and distribution of the article with the strict proviso that no changes or edits are made and the original work is properly cited (including links to both the formal publication through the relevant DOI and the license). See: https://creativecommons.org/licenses/by-nc-nd/4.0/.

\section{References}

1. Li Z, Huang Y. Application progress of acupuncture in the sequelae of stroke. Journal of Chronic Diseases 2019;20:835-7.

2. Zhang T. Chinese Stroke Rehabilitation Treatment Guidelines (Complete Edition 2011). Chinese Medical Frontiers: Electronic Edition 2012;4:55-76.

3. Yu S. Clinical Observation of Buyang Huanwu Decoction Plus Acupuncture and Moxibustion for Treatment of Stroke Sequelae. China Contemporary Medicine 2016;23:175-8.

4. Tan J, Yang Y, Li L, et al. Effects of traditional Chinese medicine packaging and acupuncture on neurological function of stroke sequelae. Guangming Traditional Chinese Medicine 2018;33:1758-60.

5. Xue M. Recent clinical application of Buyang Huanwu Decoction. Chinese and Foreign Medical 2008;25:96-7.

6. Lei H, Chen J, Zhang W, et al. Buyang Huanwu Decoction combined with acupuncture to treat sequelae of stroke. Jilin Journal of Traditional Chinese Medicine 2017;37:835-7.

7. Lei Z. Analysis of clinical efficacy of acupuncture combined with Buyang Huanwu Decoction in treating sequelae of stroke. Inner Mongolian Traditional Chinese Medicine 2018;37:74-5.

8. Liu X. Clinical effect analysis of Buyang Huanwu Tang Jiawei combined with acupuncture on stroke sequelae. Chinese Community Physician, 2019;35:108, 112.

9. Shang F, Zhu W, Sun X. Commercial Development Department. Analysis of the clinical effect of Buyang Huanwu Decoction on the sequelae of stroke. China Modern Doctor 2014;52(15):82-4. 
10. Song X. Clinical observation of acupuncture combined with Buyang Huanwu Decoction in treating sequelae of stroke. Guangming Traditional Chinese Medicine 2017;32:550-1.

11. Zhang R. Observe the therapeutic effect of Buyang Huanwu Decoction combined with acupuncture in the treatment of sequelae of stroke. Chinese Community Physicians 2016;32:119, 121.

12. He B. Buyang Huanwu Decoction combined with acupuncture to treat 50 cases of stroke sequelae. Chinese Folk Medicine 2017;26:99-100.

Cite this article as: Zeng MJ, Li LJ, Wu ZQ. Chinese herbal medicine Buyang Huanwu Decoction combined with acupuncture to treat sequela of apoplexy: a meta-analysis of randomized controlled trials. Ann Palliat Med 2021;10(2):16851692. doi: 10.21037/apm-20-1092
13. Higgins JPT, Green S. Cochrane intervention system review manual. Cochrane cooperation. Version 5.1.0 (updated in March 2011). Available online: http:// handbook.cochrane.org

14. Zhao X. The clinical efficacy of Buyang Huanwu Decoction in treating 40 patients with ischemic stroke. China Pharmaceutical 2014;23:16-8.

15. Yang GY, Luo H, Liao X, et al. Chinese herbal medicine for the treatment of recurrent miscarriage: a systematic review of randomized clinical trials. BMC Complement Altern Med 2013;13:320. 